\title{
Algunas consideraciones históricas sobre la regulación del aborto en Colombia*
}

\author{
Piedad del Valle Montoya ${ }^{* *}$ \\ Recibido: 15 septiembre de 2016 \\ Enviado a pares evaluadores: 20 de septiembre de 2016 \\ Aprobado por pares evaluadores: 9 de noviembre de 2016 \\ Aprobado por Comité Editorial: 23 de noviembre de 2016 \\ DOI: 10.22395/csye.v5n10a7
}

\section{RESUMEN}

En este artículo se busca mostrar que la conducta de quienes realizan o permiten que se realice aborto está regulada por muchos factores como las creencias religiosas, las creencias culturales y muchas veces, por el Estado, quien suele regular sus consecuencias, especialmente las penales. El texto se centra en la regulación del Código Penal colombiano de 1890 y su articulación con la medicina legal, vigente durante un período muy tradicional del país toda vez que este estuvo gobernado por el grupo político conocido como La Regeneración. Este trabajo muestra que la punibilidad del aborto responde a algunos intereses históricos.

Palabras clave: aborto; Colombia; historia; regulación.

Artículo resultado de la investigación: "El poder del saber médico en el proceso de medicalización de la Justicia en Antioquia, 1887-1914", tesis de Maestría de su autora, dirigida por Jorge Márquez Valderrama, de la Universidad Nacional de Colombia, sede Medellín.

** Abogada de la Universidad de Medellín. Magíster en Historia de la Universidad Nacional de Colombia, sede Medellín. Ha escrito libros y artículos en revistas nacionales e internacionales. Actualmente es investigadora independiente. Correo electrónico: piedadelvalle@yahoo.com 


\section{Some historical considerations on the regulation of abortion in Colombia}

\section{ABSTRACT}

This article aims to show that the behavior of those who perform or allow abortion is regulated by many factors such as religious beliefs, cultural beliefs and, often, by the State, who usually regulate their consequences, especially penal ones. The text focuses on the regulation of the Colombian Penal Code of 1890 and its articulation with legal medicine, in force during a very traditional period of the country since it was ruled by the political group known as Regeneration. This work shows that the punishability of abortion responds to some historical interests.

Keywords: abortion; Colombia; history; regulation. 


\section{Introducción}

En la historia del cristianismo no existe consenso sobre el aborto. Según la filósofa Elizabeth Badinter apenas en los siglos XII y XIII la iglesia condenó el aborto (1991). Que el catolicismo, una iglesia cristiana, ha tenido distintas posturas frente al aborto lo corrobora Mundigo quien afirma que solo lo condenó expresamente en el siglo XIX, cuando se presentó un gran cambio en la posición de la Iglesia a través de la encíclica Apostolicae Sedis, del Papa Pío IX, donde se entendió el aborto como homicidio (2005). Señala el mismo autor que:

[...] las religiones representan sistemas coordinados de creencias y prácticas que definen lo sagrado -esto es, prescriben un orden sobre ciertos fenómenos o elementos cuya existencia tiene lugar en un más allá, fuera de la vida común. Lo sagrado es a menudo colocado en un plano inaccesible o prohibido- de esto se engendran creencias y prácticas que se enlazan unas con otras formando una comunidad moral única...El propósito de las religiones es reglamentar la relación de sus adherentes con lo que se define como sagrado y con un contexto espiritual, a menudo representado por uno o varios dioses (Durkeim, citado por Mundigo 2005).

Existen, según Mundigo, variadas posiciones en las iglesias protestantes, pero en la segunda parte del siglo XX, algunas comienzan a aceptar el derecho de la mujer a seguir su propia conciencia en sus decisiones con respecto a la fecundidad, incluyendo el aborto (2005).

En asuntos religiosos, el budismo tiene otras consideraciones y existen varias posturas. El sacerdote Juan Masía observa que a algunos occidentales les extrañaba "la compatibilidad de la compasión budista con la permisividad social hacia el aborto" en Japón (La Verdad, 2009). El mismo autor precisa:

[...] Mizuko [ritual] viene de Mizu: agua y Ko: niño. Es la criatura abortada, ya sea espontánea o intencionadamente. Percibido el feto como viviente, su vida se considera flotante, a la espera de otro renacer. En el cultivo sericícola del XVII, los agricultores se deshacían de los gusanos de seda inservibles colocándolos sobre esterillas de paja flotantes en el río. Con ese imaginario de fondo, el feto abortado flotaría de regreso al mar originario de la vida. Para ritualizar el duelo, se presentan ofrendas y plegarias al bodisatva Jizô, imagen monacal e infantil, de quien se espera interceda para disculpar a la madre, y por el feto, para que cruce de nuevo el río del más allá y renazca ...La cosmovisión budista ve nacimiento y muerte, no como eventos puntuales, sino como tránsitos en un proceso: nacer es ir saliendo del mundo búdico al humano; morir es ir saliendo de éste para retornar a aquél...Nacer y morir se percibían como caminos de transición entre esta y la otra orilla. No es extraño que se aplique al feto abortado el verbo Kaeru (regresar), cuya forma transitiva sería Kaesu (devolverlo al origen) (La Verdad, 2009).

\section{Para Philip Kapleau}

[...] A diferencia de la teología lineal del oeste, el budismo enseña que la vida y la muerte presentan la misma continuidad cíclica que se observa en todos los aspectos de 
la naturaleza...La aseveración de que nada precede al nacimiento o sigue a la muerte es considerada una realidad por aquellos cuyo enfoque hacia la vida es de un aquí y un ahora; empero esta creencia...resulta absurda desde el punto de vista oriental (1990, p. 23).

Con relación al judaísmo, por ejemplo, Mundigo opina que grupos de judíos reformistas aceptan el aborto, pero sujeto a una serie de circunstancias (2005).

De manera que la comprensión del aborto, en muchos casos, es un asunto vinculado a la religión. Mundigo afirma que: "Al establecer lo sagrado, las religiones, por consiguiente, definen también lo profano, esto es las conductas y prácticas prohibidas dentro de sus códigos morales. Las religiones a través de múltiples prohibiciones ejercen una enorme influencia sobre las conductas de las personas" (2005).

Cuando se revisan otras culturas como las indígenas se observan importantes diferencias, y así, los cuna toleraron la práctica del aborto en su comunidad durante el siglo XVIII (Peralta, 2012). Los mapuches también permitían el aborto (Mora citado por De Amézquita, 2015). En las comunidades de nativos americanos se practicaba el aborto y a veces acudían al uso de plantas abortivas (Schindler\& Jackson, 2002). El mismo Masía afirma una percepción cultural religiosa en la tolerancia a la práctica del aborto japonés durante el siglo XVII (La Verdad, 2009). Como dice Mundigo, esos argumentos "tienen una larga historia y están entre-lazados con las raíces culturales de muchos pueblos (2005).

Hasta aquí se ha mostrado que frente al aborto las religiones y las culturas no tienen una posición única. Tampoco la tiene el Estado que, en muchas oportunidades, regula y ha regulado esta conducta. Denomínese Estado, sistema político o de cualquier otra forma, esta institución suele determinar las consecuencias jurídicas de la conducta abortiva, conforme a su sistema jurídico. Con razón Olmo afirma que existen distintos sistemas de control social y por esto "la pena con su propósito de prevención general positiva, contribuye a la confirmación y el aseguramiento de los otros sistemas de control social" (2005).

Ahora bien, la enorme importancia de la medicina "científica" durante los siglos $\mathrm{XIX}^{13}$ y XX posibilitó al Estado colombiano no solo la regulación de la práctica médica ${ }^{24}$, sino consagrar una singularidad en la ley penal, fundamentada en esta práctica. La práctica médica fue regulada en forma expresa en

Según Foucault, la medicina [científica] se practicó en Francia a finales del siglo XVIII y comienzos del XIX (1966).

2 La práctica médica fue regulada en varias oportunidades y la ley establecía expresamente las condiciones, especialmente de tiempo y lugar, en las cuales podían ejercer su profesión quienes no tenían título. Se pueden consultar algunas normas: Decreto 592 de 1905; ley 83 de 1914; ley 67 de 1920; Ley 39 de 1920; Ley 85 de 1922; Ley 35 de 1929 y Ley 67 de 1935, entre otras. 
Colombia en el siglo XX. Pero se puede afirmar que su reglamentación empezó en el siglo XIX, con esta salvedad en el orden penal. En este caso concreto se estudia el aborto en el Código Penal colombiano de $1890^{35}$ y su relación con la medicina legal. Es de anotar que este Código Penal estuvo vigente hasta 1938, momento en el que empezó a regir un nuevo Código Penal.

Se resalta el silencio de la Iglesia Católica frente a una contradicción precisada por una norma legal: el llamado Código Penal de 1890 señalaba explícitamente que el aborto era rechazado por la iglesia, pero al mismo tiempo regulaba algunas prácticas relacionadas con esta conducta, entre ellas, la médica. Además, la vigente Constitución Nacional de 1886 en su artículo 38 le otorgó carácter dominante a la religión católica. Estos hechos se produjeron en un momento particularmente tradicionalista en la historia del país.

Resulta importante señalar que no se conocen trabajos históricos en los que se articulen el derecho y la medicina legal en el tema del aborto en los códigos penales colombianos, especialmente en el llamado Código Penal de 1890.

\section{La situación de la medicina en Colombia a finales del siglo XIX}

En Colombia, la ley fue un acontecimiento determinante en la legitimación de la medicina "científica" en los comienzos del siglo XX. La medicalización del oficio de médico implicó un conjunto de estrategias, que terminaron por imponer la medicina "científica". En este conjunto, la normativa legal posibilitó el cambio de prácticas. La concurrencia de algunas prácticas médicas en el cambio de los siglos XIX al XX muestra a contraluz, motivaciones políticas al hacer de una de ellas la práctica médica dominante.

Los procesos económicos y sociales impactaron la concurrencia de las prácticas médicas al establecer su modulación. Esto significa que, según Ibáñez, las prácticas empíricas fueron dominantes ${ }^{46}$ en los comienzos del siglo XIX (1882). Además, y según del Valle, las prácticas empíricas tuvieron una lucha significativa con las prácticas médico-"científicas" en la segunda parte del siglo XIX, y las prácticas médico- "científicas" empezaron a ser dominantes durante las primeras décadas del siglo XX (2010).

Autores como Márquez, García \& del Valle encuentran que la medicina occidental es solo un tipo de medicina entre muchas (2012). Esta medicina se conoce

3 Bernate retoma una reconocida crítica en el sentido de que el Código Penal de 1890 era una "colcha de retazos" que tenía las incongruencias del Código Penal colombiano de 1837 (Cancino citado en Bernate).

4 Villamizar afirma sobre Cartagena y Santafé, que entre 1752 y 1802 los "cirujanos" se mantuvieron como "trabajadores artesanales sin formación académica específica" y en número superaron a los médicos. Villamizar, C. V. Los médicos invisibles: la práctica médica en Cartagena y Santafé 1752-1802. Bogotá. Tesis para optar al título de historiador. Universidad Nacional de Colombia. 2002. 
con distintas connotaciones como occidental, académica, universitaria, tradicional y "científica", entre otras, cuyas prácticas en algunos momentos fueron concurrentes en el país. La expresión "concurrente" remite al hecho, acreditado en varias investigaciones como la antes citada de Márquez, García \& del Valle, según la cual la medicina "científica", que ellos denominan "universitaria", se encontraba en competencia con otros tipos de medicina (2012). En la primera parte del siglo XIX fueron socialmente muy importantes prácticas médicas como las de curanderos, comadronas, teguas y charlatanes. Emilio Robledo refiere que en los comienzos del siglo XIX predominaban estas prácticas (1924). Pero los médicos que practicaban la medicina "científica" mostraron aceptación y rechazo frente a los otros tipos de medicina de su tiempo. Esta tensión entre las prácticas médicas es visible en las publicaciones médicas como en el escrito del médico "científico" Juan B. Londoño, publicado en la reconocida revista médica "Anales de la Academia de Medicina de Medellín" denominado "La medicina en Antioquia" donde muestra la concurrencia de las prácticas (1896).

La reglamentación en Colombia de la medicina legal, especialidad de la medicina "científica", se presentó durante el siglo XX (del Valle, 2010). Conforme a Márquez, en este debate ya "se perciben proyectos y técnicas de gobierno en los cuales la medicina ["científica"] se volvió una herramienta imprescindible de control y conocimiento de la población" (2005). Es de anotar que, según del Valle, "durante las últimas décadas del siglo XIX y principios del XX, en Colombia se conocían los grandes temas que estudiaba la medicina científica" (2010, p. 136).

Según el médico Leoncio Barreto, por lo menos desde 1890 los médicos se incluían como auxiliares de la justicia (1891). Lo cierto es que para esa fecha varios dictámenes médico legales ${ }^{57}$ obran en los procesos, y según del Valle, "la práctica se convirtió en especialidad, por lo menos hasta la segunda década del siglo XX" (2010, p. 141). Pero es necesario precisar que el artículo 65 de la Ley 100 de 1892 es, según del Valle, "la norma que empezó a instaurar en el orden legal el proceso medicalizador de la justicia en Colombia, al dejar en manos de los médicos oficiales la práctica de la prueba pericial médica" (2010, p. 151).

La medicalización ${ }^{68}$ permitió la regulación de la medicina, que en Colombia se realizó en buena medida a través de la ley. En muchas oportunidades se observa la coexistencia de las viejas prácticas médicas y las prácticas de una medicina que puede denominarse de distintas maneras, pero que es fundamentalmente

\footnotetext{
Son dictámenes fundamentados en las reglas de la medicina legal como el rendido por el médico Andrés Posada Arango en 1890 dentro del proceso 2171 del A. H. J. M. (Archivo Histórico Judicial de Medellín en el Laboratorio de Fuentes Históricas de la U. N., sede Medellín).

6 Edgardo Castro afirma que este es un tipo de poder, mediante el cual se normalizan las poblaciones y los individuos a través de la medicina. Castro Edgardo. El vocabulario de Michel Foucault. Un recorrido alfabético por sus temas, conceptos y autores. Buenos Aires: Universidad Nacional de Quilmes, 2004, p. 242.
} 
"científica", conforme se entendía este proceso durante el siglo XIX y gran parte del siglo XX. En Colombia muchas leyes muestran un zurcido entre las prácticas; entre una población acostumbrada a los empíricos y el lugar social que deseaban los titulares del nuevo saber. Pero según del Valle, el conjunto legal buscó reducir el campo del saber empírico y lego (por lego entiende a esos mismos empíricos aceptados por el poder judicial) y afianzar la categoría reciente de médico con diploma otorgado por una institución avalada por el Estado (2010).

\section{El Código Penal colombiano de 1890}

En la última parte del siglo XIX, se presentaron dos importantes acontecimientos: por un lado, se expidió la Constitución Nacional de $1886^{79}$ y, por otro lado, la medicina "científica" se empezó a imponer después de una compleja lucha con las otras prácticas médicas. Al final del siglo XIX, la medicina legal es uno de los elementos que ya se observa en algunos procesos judiciales. Entre tanto, el Código Penal de 1890, declaró que no eran punibles algunas conductas que constituían aborto. En su horizonte, la dinámica política ya había establecido su norte. Según Monroy:

[...] Regeneración no fue sólo una palabra, fue la manera más acertada de llegar a la población, de crear un lenguaje político que permitiera fomentar unas ideas y prácticas que cambiaran los destinos de la Nación a finales del siglo XIX. El proyecto regenerador se convirtió en la bandera de conservadores y liberales independientes, que unidos formaron el Partido Nacional y utilizaron el lema "Regeneración o Catástrofe", para señalar y aplacar a los liberales radicales y su proyecto de federalismo, regido por la Constitución de 1863, llamada también la Constitución de Rionegro. El discurso de los conservadores de la época se preocupó por dejar clara su pretensión de cambiar el país, de poner todo en orden, de transformar y educar a sus ciudadanos... (2012, p. 222).

Obregón precisa que el objetivo era no solo construir un "Estado centralizado, sino garantizar la "paz científica" como decía Núñez, haciéndose eco del movimiento cientificista". Por esta razón, las medidas contra la libertad de prensa "no implicaron represión contra las publicaciones científicas" (1992, p. 7). Según Monroy:

[...] La construcción de la Nación no solo se pretendió a través del establecimiento de leyes y normas como la constitución de 1886 o el concordato de 1887, entre las más significativas, aquí fue fundamental la imposición de valores tradicionales y morales y de una cultura particular, que cobijó a toda la población sin distinción de raza, lengua, religión, territorio, etcétera. Simplemente aquellos, que no aceptaban este proyecto o asumían estos valores, eran considerados como "salvajes" a los cuales era necesario "civilizar", todo esto en nombre de la Nación (2012, p. 228).

$\overline{7}$ Con esta constitución, las leyes son nacionales. 
Además, dice la misma autora que "La Constitución privilegió el deseo de los conservadores y de la iglesia para establecer en las normas legales, que la religión católica era de la Nación y que la iglesia tendría amplio [poder] en ciertos aspectos de la vida nacional" (Melo citado por Monroy 2012, p. 229). Este proyecto y su constitución "buscaban transformar a la población en el ámbito moral y cultural bajo la influencia de la religión católica y las relaciones iglesia-Estado" (2012, p. 223). Expedida la Constitución Nacional de 1886 se adoptaron algunos códigos y se procedió a la unificación de la legislación nacional, mediante la Ley 57 de 15 de abril de 1887. Desde muy temprano se dijo que el llamado Código Penal de 1890 era casi el mismo de 1837, vigente en 1858 en el antiguo Estado de Cundinamarca, con algunos ajustes, que se justificaron inicialmente, según Cárdenas et al., "en el nuevo cambio de Constitución y en las nuevas exigencias científicas". Sin embargo, afirma el autor que no se presentaron las modificaciones anunciadas (1938, p. 5). (Negrilla fuera del texto).

A pesar de que en la Constitución Nacional se privilegió a la religión católica, y en su artículo 38 señaló que era la de la "Nación", en la comprensión penal de las conductas constitutivas de aborto fueron determinantes "las exigencias científicas", entre ellas, la legitimación legal de la práctica médica "científica". Ahora bien, el Código Penal de 1890 reguló el delito de aborto en los artículos 638 a 643 y consagró una exención de pena en el artículo 640. Dice Rodríguez:

[...] No se incurrirá en pena alguna cuando se procure o efectúe el aborto como un medio absolutamente necesario para salvar la vida de una mujer, ni cuando en conformidad con los sanos principios de la ciencia médica, sea indispensable el parto prematuro artificial". La norma agrega que se limita a eximir de la pena "al que con rectitud y pureza de intenciones se cree autorizado para ocurrir a dichos medios. No por eso debe creerse que la ley aconseja el empleo de esos medios, que generalmente son condenados por la Iglesia. Únicamente se limita a eximir de pena al que con rectitud y pureza de intenciones se cree autorizado para acudir a dichos medios" (1927, p. 876). (Negrilla y resalto fuera del texto).

De manera que el texto legal exime de pena al médico "científico" y considera su actuación realizada con rectitud y pureza de intenciones. Esta misma comprensión tenía la medicina legal y, si bien es cierto que Barreto cita artículos diferentes, el estado de la especialidad es el mismo.

[...] Para saber hasta donde alcanza la facultad de un médico para producir el aborto, es necesario resolver muchas cuestiones médicas de grande importancia; pero que todas deben estar basadas sobre las probabilidades de vida para la mujer por el estado de su constitución y la naturaleza del accidente que quiere remediarse; y por la viabilidad del feto...no hay duda que es la mujer la única que tiene derecho a la vida y a quien debe tratar de salvarse, produciendo el aborto....pero cuando el feto es viable y sobre todo cuando ya está cerca el término de la preñez, si el médico se encuentra 
al frente de uno de esos casos en que no hay otro medio que sacrificar al feto para poderlo extraer ó practicar la operación Cesárea que equivale casi tanto como á sacrificar á la mujer: sólo podrá decidirse la cuestión, decidiendo cuál de los dos es el que tiene más probabilidades de vivir (Barreto, 1891, p. 56). (Negrillas fuera del texto).

Lo anterior significa que médicamente se podía practicar el aborto hasta el noveno mes de la preñez, antes del nacimiento. El médico legista lo manifiesta en forma expresa: "Para los tocologistas, el aborto es "la expulsión del feto antes de su viabilidad"; pero para los médicos jurisconsultos debe ser "la expulsión del feto antes del término natural de la preñez", conforme a nuestros Códigos"”. (Barreto, 1891, 52). Continúa Barreto ilustrando cuando ha habido tentativa de aborto: "averiguando si los medios que se dice haber empleado lo han sido por una persona extraña a la ciencia, ó por un facultativo, en cuyo caso debe comprobarse la científica y precisa indicación de su empleo (1891, p. 54). (Resalto fuera del texto).

Sin pronunciarse sobre cuál de las vidas prevalece, el médico legista, doctor Carlos E. Putnam, publica en 1896 su reconocido tratado médico legal y citando correctamente el articulado del Código Penal de 1890, afirma que:

[...] Para la ley penal es aborto todo mal parto provocado con mal fin, haya o no llegado el feto a la edad en que puede vivir... mientras la ley abraza, además, la intención y la voluntad de su autor, el fin con que se ejecuta, sea cualquiera la época de la preñez (Putnam, 1896, p. 313). Negrilla fuera del texto.

Articulando la norma legal a los análisis de Barreto y Putnam se puede afirmar que la profesión médica "científica", durante la vigencia del Código Penal de 1890, que se extendió hasta 1938, podía practicar el aborto en cualquier tiempo de la preñez por razones médicas. El Código Penal de 1936 también le daba mucha importancia al carácter científico de la medicina, pero con otros énfasis. Este hecho se puede apreciar en un título de su tiempo: El nuevo Código Penal y el modo de interpretarlo científicamente, de Edmundo Vilar.

Para entender la vigencia del artículo 640 del Código Penal de 1890 es necesario tener en cuenta no solo la mayúscula importancia del carácter científico de la medicina en este momento histórico, sino el hecho fundamental de que la Iglesia católica, como dice Mundigo, empezó a endurecer su posición respecto al aborto desde 1917, cuando extendió su reprobación a médicos y enfermeras que hubieran participado en dicha práctica (2005). Es necesario insistir en que la Iglesia católica no ha tenido una posición única frente al aborto (por distintas razones) en su larga historia, y el endurecimiento de su posición es muy reciente. Las afirmaciones de Mundigo en el sentido de que la dura posición de la Iglesia es muy reciente son confirmadas por Hurts (1998). En el mismo sentido se pronuncia Sagot quien afirma: 
[...] Una de las realidades que es necesario conocer, y que este debate ha dejado intencionalmente en la sombra, es la historia de las ideas de la Iglesia Católica sobre el aborto. En la actualidad conocemos una posición muy estricta y contundente, y pareciera que ésta ha sido siempre su posición oficial. Sin embargo, las concepciones en tomo a la interrupción voluntaria de un embarazo han cambiado a lo largo de la historia de la Iglesia Católica.

$\mathrm{Al}$ analizar esta historia, quizás lo más sorprendente es que siendo este un tema de tanta relevancia para el Vaticano, -que ha motivado la movilización de las fuerzas católicas de todo el mundo para impedir acuerdos mundiales sobre población, salud y derechos de las mujeres-, la doctrina sobre el aborto no está gobernada por la Infalibilidad Papal y, por tanto, no es materia de dogma de fe. Es sorprendente también que la posición, actual del Vaticano que establece la existencia de un ser humano desde la concepción, no haya sido un criterio dominante históricamente, sino que lo es tan sólo desde 1869. En efecto, anterior a esta fecha los debates internos en la Iglesia Católica no han girado únicamente ni principalmente en torno a esta tesis (2002).

De manera, que el carácter científico que notoriamente ostentaba la medicina en el siglo XIX termina por imponerse en la codificación penal colombiana de esa época. La importancia del carácter científico y paradigmático de la medicina en la cultura europea del siglo XIX la recuerda Foucault:

[...] Si las ciencias del hombre han aparecido en el prolongamiento natural de las ciencias de la vida, no es porque ellas estaban biológicamente subtensas, sino médicamente: se encuentra en su estructura de origen una reflexión sobre el hombre enfermo, y no sobre la vida en general, una reflexión presa en un problema de división más que en un trabajo de unificación, e íntegramente ordenada para el emparejamiento de lo positivo y de lo negativo (1966, p. 62).

El pensador francés recalca la importancia de la medicina en la constitución de las ciencias del hombre, en su metodología y hasta en su ontología, ya que el ser del hombre es objeto de un saber positivo, posibilidad que hace al hombre sujeto y objeto de su propio conocimiento; por esto dice que "el pensamiento médico está comprometido por derecho propio en el estatuto filosófico del hombre" (Foucault, 1966, p. 278).

Volviendo al Código Penal es necesario afirmar que el artículo 642 de esta normativa consagró la concurrencia de las prácticas. Según Rodríguez:

[...] Pero si fuere mujer honrada y de buena fama anterior y resultare, a juicio de los jueces, que el único móvil de la acción fue el de encubrir su fragilidad, se le impondrá solamente la pena de tres a seis meses de prisión, si el aborto no se verifica; y de cinco a diez meses, si se verifica (1927, p. 877).

Pues bien, el poder judicial en varias oportunidades aplicó favorablemente algunas de las circunstancias relacionadas en los artículos 117 a 119 del Código Penal de 1890 correspondientes al estatuto de agravantes y atenuantes de la 
ley penal, pero en múltiples oportunidades las fallas probatorias, entre ellas la falta de un dictamen médico legal o la deficiencia en las formas jurídicas ${ }^{810}$, posibilitaron a los funcionarios dictar múltiples sobreseimientos ${ }^{911}$. Los jueces penales se centraban en practicar las pruebas como lo acreditan muchos procesos y estas tenían la fuerza que brindaban las normas del enjuiciamiento criminal, reglamentado en el Código Judicial.

\section{Conclusión}

Muchos factores impactan la regulación penal del aborto como las religiones, una cultura determinada y, en muchas oportunidades, el Estado, que suele regular sus consecuencias, especialmente las penales. Es cierto que el Código Penal colombiano de 1890, que es el objeto de este estudio, consagró el aborto punible, pero la dinámica que tiene la llamada "medicina científica" muestra que la sanción a esta conducta es histórica, es decir, que se dan cambios en la concepción del aborto que hacen que una conducta sea sancionada o no y este hecho tiene incidencia política.

En este caso quedó acreditada la importancia científica a pesar de la alianza estatal con la religión católica en un momento histórico determinado en Colombia: la práctica de la medicina "científica" se estaba imponiendo en el país. La ley fue una aliada estratégica en el relevo de otro tipo de prácticas médicas y este hecho se extendió a algunos de los modelos penales. El análisis del mismo Código Penal de 1890 y de literatura producida durante este período, especialmente de medicina legal, muestra cómo la medicina representa una perspectiva presente en esta decodificación.

El silencio de la Iglesia católica en un momento en que era particularmente poderosa y cuando el país era muy tradicionalista extiende preguntas no solo respecto a la importancia de la medicina y a su dinámica política, sino al proceso de medicalización que estaba desplegándose en el país. Es de anotar que este código estuvo vigente hasta 1938, fecha en la que entró a regir el Código Penal de 1936. Cuando se piensa que los problemas sociales en torno a este modelo punible impactan algunas decisiones políticas, se entiende su importancia.

\section{Referencias hibliográficas}

Badinter, E. (1991). ¿Existe el instinto maternal? Historia del amor maternal. Siglos XVII al XX. Barcelona: Paidós.

8 "Se refiere a los procedimientos aplicables a cada delito..." Del Valle, P., Amores criminales. Un caso de parricidio en Colombia, 2015, p. 198.

9 El sobreseimiento consistía en que el juez declaraba judicialmente que no había lugar al seguimiento de la causa por falta de pruebas. Rodríguez, 1927, p. 1258. 
Barreto, L. (1890). Prontuario de Medicina legal y jurisprudencia médica. Bogotá: Imprenta La Comercial.

Bernate, Francisco. El Código Penal colombiano de 1890. Estudios Socio-Juríd. Vol 6. N. 2. Bogotá. Julio/diciembre 2004.

Cárdenas, P. et al., (1938). "Exposición de motivos" de la Comisión de Asuntos Penales y Penitenciarios, Código Penal, Leyes 95 y 205 de 1936, artículo 13 de la Ley 124 de 1937 y Decreto Legislativo 2300 de 1936, Código de Procedimiento Penal, Ley 94 de 1938 y Decretos Legislativos números 111111699 de 1938. Bogotá: Imprenta Nacional.

Castro, E. (2004). El vocabulario de Michel Foucault. Un recorrido alfabético por sus temas, conceptos y autores. Buenos Aires: Universidad Nacional de Quilmes.

De Améstica, C. (2015, 2 de marzo). El aborto en el mundo Mapuche es una responsabilidad exclusivamente femenina. La Segunda. Recuperado de 2015-03-02 | 11: Crónica $\mid$ A (L42L06R2) - La Segunda

Del Valle, P. (2010). La medicalización de la justicia en Antioquia (1887-1914). Medellín: Editorial Universidad de Antioquia.

Del Valle, P. (2015). Amores criminales. Un caso de parricidio en Colombia. En Microhistorias de la transgresión. Hering, M. \& Rojas, N. (edit.). Bogotá: Universidad Nacional de Colombia, sede Bogotá, Universidad Cooperativa de Colombia y Universidad del Rosario.

Documento N. 2171 del A. H. J. M. (Archivo Histórico Judicial de Medellín en el Laboratorio de Fuentes Históricas de la U. N., sede Medellín).

Foucault, M. (1966). El nacimiento de la clínica. Una arqueología de la mirada médica. México: Editorial Siglo XXI.

Hurts, J. (1998). La historia de las ideas sobre el aborto en la Iglesia católica. Lo que no fue contado. México. Disponible en: http://repositorio.gire.org.mx/bitstream/123456789/2358/1/libro3.pdf

Ibáñez, P. M. (1882, junio 24). Memorias para la historia de la medicina en Santafé. Revista Médica de Bogotá. Vol. VII, pp. 5-23.

Kapleau, P. (1990). La rueda de la vida y la muerte. Una guía práctica y espiritual. México: Árbol Editorial S. A. de C. V.

Londoño, J.B. (1896, octubre 2) La medicina en Antioquia. Anales de la Academia de Medicina de Medellín. Vol. VIII, pp. 81-86.

Márquez, J. (2005). Ciudad, miasmas y microbios. La irrupción de la ciencia pasteriana en Antioquia. Medellín: Universidad Nacional de Colombia, sede Medellín. Editorial Universidad de Antioquia.

Márquez, J., García, V. y Del Valle, P. (2012, septiembre 23). La profesión médica y el charlatanismo en Colombia en el cambio del siglo XIX al XX". Quipu. Recuperado de http://www.academia. edu/10287250/La_profesi\%C3\%B3n_m\%C3\%A9dica_y_el_charlatanismo_en_Colombia_en_el_cambio_del_siglo_XIX_al_XX

Masía, J. (2009, 28 de julio). Rito japonés, tras el aborto. La verdad.es Recuperado de http://www. laverdad.es/murcia/prensa/20090728/opinion/rito-japones-tras-aborto-20090728.html

Monroy, C. L. De federalismo a regeneración. El paso de Estados soberanos a departamentos políticoadministrativos. Boyacá, 1886-1903 (2012, febrero 9). Historelo. Revista de Historia Regional y Local. Recuperado de http://www.scielo.org.co/pdf/histo/v4n7/v4n7a09.pdf 
Mundigo, A. I. (2005, 17 de agosto) Religión y salud reproductiva: encrucijadas y conflictos. Trabajo presentado en la II Reunión de investigación sobre embarazo no deseado y aborto inseguro. México 17 a 19 de agosto. Recuperado de http://www.pasa.cl/wp-content/uploads/2011/08/Religion _ y _ salud_reproductiva._Encrucijadas_y_conflictos_Mundigo_Axe.pdf

Obregón, D. (1992). Sociedades científicas en Colombia. La invención de una tradición 1859-1936. Bogotá: Banco de la República.

Olmo, P.O. (2005, julio 19). El concepto de control social en la historia social. Estructuración del orden y respuestas al desorden. Historia Social 51. Recuperado de https://dialnet.unirioja.es/servlet/ articulo?codigo $=1421130$

Peralta, J. (2012, enero 31). Los cuna y sus saberes médicos. La "ciencia" de los "bárbaros" bajo la mirada del mundo ilustrado. Historia Crítica. DOI: http://dx.doi.org/10.7440/histcrit46.2012.04

Putnam, C. E. (1896). Tratado práctico de medicina legal en relación con la legislación penal y procedimental del país. Bogotá: Imprenta de Antonio M. Silvestre.

Robledo, E. (1924, enero 30). "La medicina en los departamentos antioqueños". Repertorio Histórico. Academia Antioqueña de Historia. Vol. 1 y 2, pp. 5-47.

Rodríguez, E. (1927). Constitución y Códigos de Colombia. Bogotá: Librería América.

Schindler, K. \& Jackson, A. E. (2002). Indigenous Women's Reproductive Rights The Indian Health Service and Its Inconsistent Application of the Hyde Amendment. USA: Lake Andes, South Dakota.

Vilar, E. (1939). El nuevo Código Penal y el modo de interpretarlo científicamente. Bogotá: Librería Colombiana Camacho Roldán \& Cía., S. A.

Villamizar, C.V. (2002). Los médicos invisibles: la práctica médica en Cartagena y Santafé 1752-1802. Bogotá. Tesis de pregrado. Universidad Nacional de Colombia. Bogotá.

Sagot M. Aborto inducido: ética y derechos en Med. leg. Costa Rica vol.19 n. 2, Heredia septiembre 2002. Disponible en http://www.scielo.sa.cr/scielo.php?script=sci _arttext\&pid=\$1409-00152002000200008 
